\title{
Prospective 3-month study on the efficacy of UV-free blue light in mild psoriasis vulgaris treatment
}

\author{
Aleksandra Lesiak, Igor A. Bednarski, Joanna Narbutt \\ Dermoklinika Centrum Medyczne, Lodz, Poland
}

Adv Dermatol Allergol 2021; XXXVIII (3): 446-449

DOI: https://doi.org/10.5114/ada.2021.107931

\begin{abstract}
Introduction: Psoriasis is one of the most common chronic skin diseases affecting up to $2 \%$ of the general population. In recent years, an important direction for the development of treatment for psoriasis has been the use of blue light emitted by LED lamps.

Aim: To evaluate the efficacy of blue-light emitting device in psoriasis vulgaris treatment.

Material and methods: The study involved 30 adults with a mild form of psoriasis vulgaris not previously receiving biological treatment and other forms of phototherapy. Participants of the study used a device emitting blue LED light for 3 months. Each participant received a device for use at home, with which he/she exposed 2 psoriatic lesions located on the limbs. Before and after the study, the severity of the disease was evaluated using PASI, DLQI and LPSI.

Results: During 3 months of the therapy, a statistically significant decrease in the mean LPSI (in both treated locations) and DLQI was noted (LPSI decrease from $5.25 \pm 1.82$ to $1.98 \pm 1.74, p<0.0001$; DLQI from $7.36 \pm 5.59$ before the study to $5.23 \pm 4.62$ after the study.

Conclusions: Our results confirm that phototherapy using blue LED light is both a safe and highly effective way to treat psoriasis.
\end{abstract}

Key words: psoriasis, blue light, phototherapy.

\section{Introduction}

Psoriasis is one of the most common chronic skin diseases affecting up to $2 \%$ of the general population [1]. Due to its chronic, recurrent and long-lasting character and stigmatizing effect, patients with psoriasis may also develop symptoms of anxiety or depression [2]. In comparison to the general population, people suffering from psoriasis are at risk of developing complications from the cardiovascular system, which also contributes to higher mortality in this group of patients [3]. Despite many years of research, determining the exact causes of psoriasis is still impossible as psoriasis is a multifactorial disease in which both genetic and environmental factors may play a role. It is generally recognized that psoriasis is an immune-mediated disease in which the main disorder is the acceleration of the transition time of keratinocytes from the basal layer to the stratum corneum (turnover time - TOT) and their increased proliferation [3]. For this reason, the treatment of psoriasis is often multidirectional and includes drugs that reduce inflammation and remove the hyperkeratotic plaques in different mechanisms of action. Phototherapy of psoriasis is one of the basic methods of treatment of mild to severe psoriasis. Despite the fact that phototherapy for psoriasis has been used in dermatology for over 70 years, we still observe a dynamic development of this method of treatment, resulting from new discoveries in the field of immunology and photobiology [4]. Basically, there are three main types of phototherapy used in the treatment of psoriasis: broadband ultraviolet B (BB-UVB), ultraviolet B (narrowband-UVB, NBUVB) therapy and a combination of psoralen with ultraviolet radiation type A (PUVA) [5]. In recent years, an important direction for the development of phototherapy for psoriasis has been the use of blue light emitted by LED lamps (light emitting diode) [6]. In studies using cell lines, it has been shown that blue light reduces the production of cytokines by dendritic cells and normalizes the proliferation of fibro-

Address for correspondence: Igor A. Bednarski MD, Dermoklinika Centrum Medyczne, 93 Kościuszki St, Lodz, Poland, e-mail: igorbednarskiv@gmail.com Received: 11.03.2019, accepted: 19.01.2020. 
Table 1. Detailed results of erythema, induration, scaliness and LPSI before enrolment into the study and after 3 months of the therapy

\begin{tabular}{|c|c|c|c|c|c|c|c|c|c|c|c|}
\hline \multirow[t]{2}{*}{ Parameter } & \multicolumn{5}{|c|}{ Before } & \multicolumn{5}{|c|}{ After } & \multirow[t]{2}{*}{$P$-value } \\
\hline & Mean & Median & $1^{\text {st }}$ quartile & $3^{\text {rd }}$ quartile & SD & Mean & Median & $1^{\text {st }}$ quartile & 3rd quartile & SD & \\
\hline Redness & 1.85 & 2.00 & 1.00 & 2.00 & 0.60 & 1.00 & 1.00 & 1.00 & 1.00 & 0.66 & $<0.0001$ \\
\hline Induration & 1.80 & 2.00 & 1.00 & 2.00 & 0.80 & 0.54 & 0.00 & 0.00 & 1.00 & 0.72 & $<0.0001$ \\
\hline \multirow[t]{2}{*}{ Erythema } & 1.60 & 2.00 & 1.00 & 2.00 & 0.93 & 0.43 & 0.00 & 0.00 & 1.00 & 0.67 & $<0.0001$ \\
\hline & & \multicolumn{4}{|c|}{ Before } & \multicolumn{5}{|c|}{ After } & \\
\hline Parameter & Mean & Median & $1^{\text {st }}$ quartile & $3^{\text {rd }}$ quartile & SD & Mean & Median & $1^{\text {st }}$ quartile & $3^{\text {rd }}$ quartile & SD & $P$-value \\
\hline Redness & 1.85 & 2.00 & 1.00 & 2.00 & 0.60 & 1.00 & 1.00 & 1.00 & 1.00 & 0.66 & $<0.0001$ \\
\hline Induration & 1.80 & 2.00 & 1.00 & 2.00 & 0.80 & 0.54 & 0.00 & 0.00 & 1.00 & 0.72 & $<0.0001$ \\
\hline Erythema & 1.60 & 2.00 & 1.00 & 2.00 & 0.93 & 0.43 & 0.00 & 0.00 & 1.00 & 0.67 & $<0.0001$ \\
\hline
\end{tabular}

blasts and keratinocytes [7-9]. These observations have been confirmed in a number of clinical trials using a homeuse device that emits blue light using LEDs [10]. Until now, blue light therapy has been used to treat acne, neonatal jaundice (NJ), and Crigler-Najjar syndrome. Nevertheless, long-term studies on the safety of blue light in psoriasis are missing.

\section{Material and methods}

\section{Study group}

The study involved 30 adults - 15 males and 15 females (mean age: $47.20 \pm 15.23$ ) with a mild form (PASI $<10$ ) of psoriasis vulgaris (mean PASI: $5.17 \pm 2.0$ ) not previously receiving biological treatment and other forms of phototherapy. The study population comprised of individuals with Fitzpatrick's skin phototypes I-III (5 individuals with phototype I, 19 with phototype II and 5 with phototype III). This study was carried out in the Dermoklinika Medical Centre in Lodz, Poland. The approval of the Bioethical Commission (No. RNN/56/15/EC) was obtained before conducting the trial. The exclusion criteria were: conditions with photosensitivity (porphyria, solar urticaria), immunosuppression, active or cured skin cancers, any therapy that uses photosensitizing drugs, uncontrolled hypertension, myocardial infarction or stroke, and atherosclerosis. Pregnant and breastfeeding women were also excluded from the study.

Participants of the study used a device emitting blue LED light (Philips, Eindhoven, Netherlands) for 3 months (once daily for 5 continuous days each week). Each participant received a device for use at home, with which he/she exposed 2 psoriatic lesions located on the limbs. Before and after the study, the severity of the disease was evaluated using PASI, Dermatology Life Quality Index (DLQI) and local psoriasis severity index (LPSI).

\section{Statistical analysis}

The differences between the groups were calculated using the Wilcoxon pair order test for dependent pairs of observations, preceded by the analysis of the normality of the group distribution (Shapiro-Wilk test). The level of statistical significance was assumed as $p<0.05$.

\section{Results}

During 3 months of the therapy, a statistically significant decrease in the mean LPSI (in both treated locations) and DLQI was noted (LPSI decrease from $5.25 \pm 1.82$ to $1.98 \pm 1.74, p<0.0001$; DLQI from $7.36 \pm 5.59$ before the study to $5.23 \pm 4.62$ after the study). Moreover, we observed a statistically significant decrease in all parameters included in LPSI score: erythema, induration and scaliness (detailed results are shown in Table 1 ). The exact group characteristics are presented in Figures 1, 2 A, B. In the study group, 4 patients achieved 100\%, 9 patients at least $75 \%$, and 7 at least $50 \%$ resolution of psoriatic lesions measured by LPSI. None of the patients reported any adverse reactions in the form of erythema and exacerbation of psoriatic lesions.

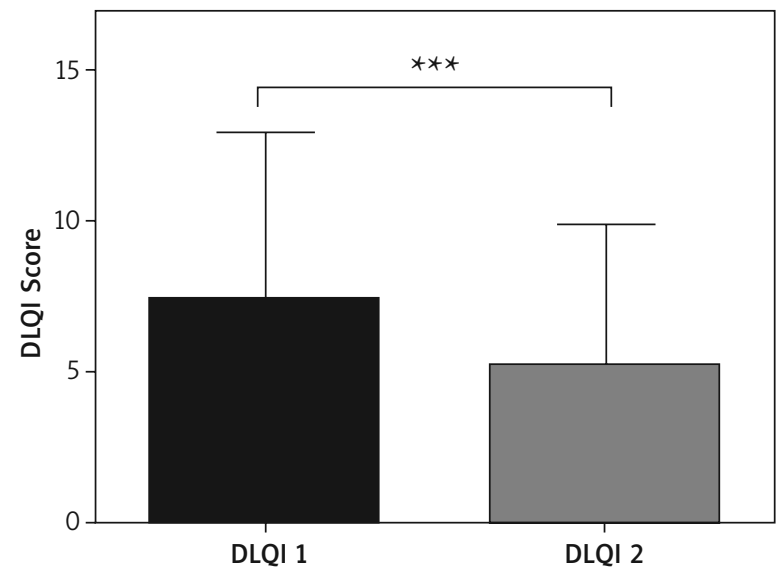

Figure 1. Changes in DLQI before and after 3 months of the therapy 
A

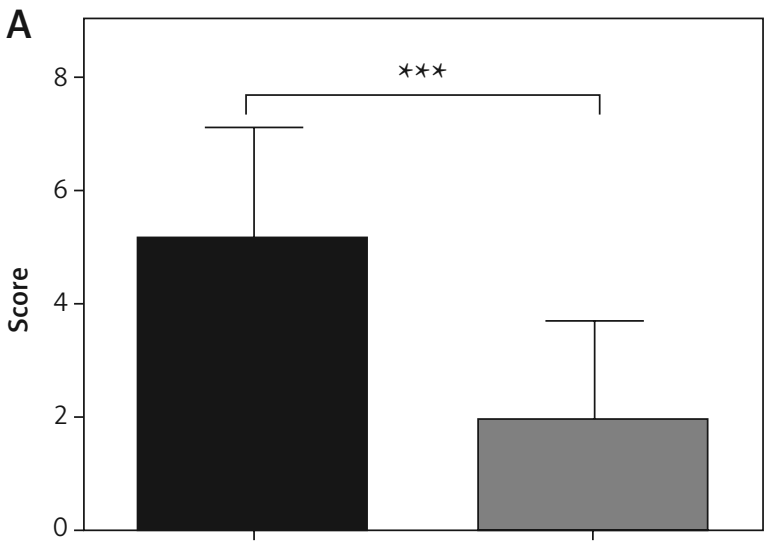

LPSI 1

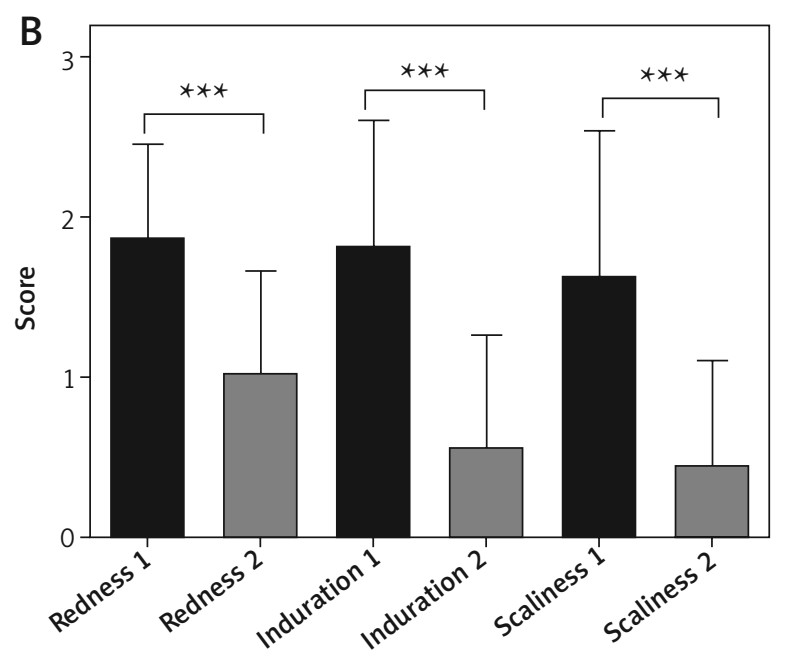

Figure 2. Changes in LPSI and LPSI parameters before and after 3 months of the therapy

\section{Discussion}

Our results confirm that phototherapy using blue LED light is both a safe and highly effective way to treat psoriasis. In the current in vitro studies, blue light has been shown to normalize the proliferation of keratinocytes, endothelial cells and fibroblasts without a cytotoxic effect [7, 11]. Liebmann et al. [7] observed that irradiation of keratinocytes with blue light at a wavelength of $453 \mathrm{~nm}$ does not show a cytotoxic effect up to high fluences, while irradiation with lengths from 412 to $426 \mathrm{~nm}$ is associated with a cytotoxic effect at relatively low fluences, thus indicating that blue light can be used in the therapy of psoriasis. In two prospective and randomized clinical trials $[6,10]$, it was observed that irradiation of psoriatic lesions with a $453 \mathrm{~nm}$ wavelength resulted in a significant reduction in the local psoriasis symptoms after only 4 weeks of treatment, moreover complete clinical remission was achieved in some patients. In contrast to our results, the comparison between the irradiated change and the control change was used for the assessment. In addition, an evaluation of pruritus using the VAS scale was used in both studies. In our study, we achieved complete clinical remission of irradiated lesions in 4 patients, without any side effects.

Increased proliferation of keratinocytes with shortening the transition time between the stratum basalis and stratum corneum as well as inflammatory infiltration from $T$ lymphocytes are phenomena typical for psoriasis. For this reason, the effects observed clinically after the end of blue light therapy can be explained by phenomena occurring at the cellular level, normalization of proliferation and differentiation of keratinocytes [7]. In addition, it has been shown that blue light can also inhibit the production of pro-inflammatory cytokines by dendritic cells [9].
However, in a study conducted by Maari et al. [12], no satisfactory clinical effect was achieved. Pfaff et al. [10] postulate that this could be due to the use of a source of light other than LED, lower energy of radiation used and a shorter duration of the test.

Our research confirms the results achieved by other research teams, but in case of a small study group also the placebo effect should be taken into account. The possibility of treating psoriasis at home, high effectiveness of therapy, lack of side effects and improvement of the patient's quality of life (measured by DLQI) make it an alternative form of treatment that should be considered in the treatment of psoriasis. Patients also considered this form of therapy as easy in application, convenient, well integrated with their everyday life. It seems that blue light therapy may be beneficial for patients, but larger studies with placebo are required to confirm the efficacy and safety of this treatment.

\section{Acknowledgments}

The authors would like to thank Mr. Gregor Jelen, prof. Matthias Born and prof. Jörg Liebmann for their continuous support and great help with the study.

\section{Conflict of interest}

The study was funded by the grant provided by Philips Light \& Health.

\section{References}

1. Almutawa F, Alnomair N, Wang Y, et al. Systematic review of UV-based therapy for psoriasis. Am J Clin Dermatol 2013; 14: 87-109.

2. Ferreira BIRC, Abreu JLPDC, Reis JPGD, Figueiredo AMDC. Psoriasis and associated psychiatric disorders: a systematic 
review on etiopathogenesis and clinical correlation. J Clin Aesthet Dermatol 2016; 9: 36-43.

3. Nestle FO. Psoriasis. N Engl J Med 2009; 361: 496-509.

4. Racz E, Prens EP. Phototherapy and photochemotherapy for psoriasis. Dermatol Clin 2015; 33: 79-89.

5. Mehta D, Lim HW. Ultraviolet B phototherapy for psoriasis: review of practical guidelines. Am J Clin Dermatol 2016; 17: 125-33.

6. Weinstabl A, Hoff-Lesch S, Merk HF, von Felbert V. Prospective randomized study on the efficacy of blue light in the treatment of psoriasis vulgaris. Dermatology 2011; 223: 251-9.

7. Liebmann J, Born M, Kolb-Bachofen V. Blue-light irradiation regulates proliferation and differentiation in human skin cells. J Invest Dermatol 2010; 130: 259-69.

8. Awakowicz P, Bibinov N, Born M, et al. Biological stimulation of the human skin applying health promoting light and plasma sources. Contrib Plasma Phys 2009; 49: 641-7.

9. Fischer MR, Abel M, Kostka SL, et al. Blue light irradiation suppresses dendritic cells activation in vitro. Exp Dermatol 2013; 22: 558-60.

10. Pfaff S, Liebmann J, Born M, et al. Prospective randomized long-term study on the efficacy and safety of UV-free blue light for treating mild psoriasis vulgaris. Dermatology 2015; 231: 24-34.

11. Taoufik K, Mavrogonatou E, Eliades T, et al. Effect of blue light on the proliferation of human gingival fibroblasts. Dent Mater 2008; 24: 895-900.

12. Maari C, Viau G, Bissonnette R. Repeated exposure to blue light does not improve psoriasis. J Am Acad Dermatol 2003; 49: 55-8. 